Proyecciones Journal of Mathematics

Vol. 30, No 3, pp. 441-457, December 2011.

Universidad Católica del Norte

Antofagasta - Chile

\title{
A Quantum Mechanical Proof of the Fourier Inversion Formula
}

\author{
NELSON N. DE O. CASTRO \\ UNIVERSIDADE FEDERAL DE PARAÍBA, BRASIL \\ RAMÓN MENDOZA \\ UNIVERSIDADE FEDERAL DE PERNAMBUCO, BRASIL \\ and \\ JACQUELINE ROJAS * \\ UNIVERSIDADE FEDERAL DA PARAÍBA, BRASIL \\ Received : March 2011. Accepted : May 2011
}

\begin{abstract}
The translation of the observable, position and momentum, of a given particle in the real line, at a certain time $t$, from Classical $\mathrm{Me}$ chanics, into the operators, position and momentum, in Quantum Mechanics, gives us the inspiration to make a proof of the existence of the Fourier's Inverse Transform, using algebraic relations involving these operators (position and momentum), a few of Linear Algebra and Analysis, without resorting to the classical technics like Fubini's Theorem and Lebesgue's Dominated Convergence Theorem.
\end{abstract}

MSC 2000 : Primary 42A38; Secondary 81Q99.

Key Words : Fourier transform, position operator, momentum operator, extension.

*Partially supported by CNPq Grant 620108/2008-8 (Edital Casadinho). 


\section{Introduction}

As commented in the abstract, the inspiration to make an accessible, essentially algebraic proof of the existence of the Fourier inverse operator, comes from the notions of position and momentum in Classical Mechanics and their corresponding operators in Quantum Mechanics. In fact, on the one hand it is known that the eigenvalues of the operators, position and momentum, (see (3.1) and (3.2) for their precise definitions) must be real numbers, since these correspond to the position and momentum of a given particle in the real line, at time $t$, but on the other hand, the position operator, $P$, (in (3.1)), does not has eigenvectors and the momentum operator, $M$, (in (3.2)), has the $\mathcal{C}^{\infty}$ - function $f_{\lambda}(x)=e^{i \lambda x}$ as an eigenvector associated to the complex eigenvalue $\lambda$. So, from a physical point of view, it is of interest to have an extension, $\mathcal{P}$, of the position operator, $P$, and to do a very careful choice of the domain for the extension, $\mathcal{M}$, of the momentum operator, $M$, in order to get only real eigenvalues for their respective extensions. With this aim we devote Section 3 to study the eigenvectoreigenvalue problem for this operators and their extensions. At this point we show in Proposition 3.1 that all real number $\lambda$ is an eigenvalue of $\mathcal{P}$, whose eigenspace, $E_{\lambda}(\mathcal{P})$, is a one-dimensional vector space.

The largest part of this paper is devoted to prove the following key equation, $\Lambda^{2}=I$, where $\Lambda$ denotes the Fourier transform operator and $I$ is "almost" the identity operator. In fact $I^{2}$ is the identity operator as can be easily seen from its definition in (4.1). At last but not least, we explore the extension of the Fourier transform to $\mathcal{L}^{2}(\mathbf{R})$ (See subsection 5.2). In particular, the existence of the inverse of $\Lambda$, (cf. Theorem 4.7), in $\mathcal{S}$, implies the existence of the Fourier inverse transform, in $\mathcal{L}^{2}(\mathbf{R})$. In the last section we also explore the extension $\mathcal{F}$, as in (2.2), of the Fourier transform $\Lambda$, in (2.1). Finally in Remark 5.3, we point out the necessary modifications which has be done to carry on the proof of the Theorem 4.7 for the Fourier transform in $\mathbf{R}^{n}$, (see (5.1)), as it was made in the one-dimensional case.

\section{Notation and Preliminary Results}

In this section we introduce the main spaces we shall deal with. From now on, we consider complex-valued functions $\varphi: \mathbf{R} \rightarrow \mathbf{C}$.

- $\mathcal{C}^{\infty}(\mathbf{R}), \mathcal{E}(\mathbf{R})$ or $\mathcal{E}$. By $\mathcal{C}^{\infty}(\mathbf{R})$ we mean the vector space of all infinitely differentiable functions $\varphi: \mathbf{R} \rightarrow \mathbf{C}$. We use the notation $\mathcal{E}$, 
instead of $\mathcal{C}^{\infty}(\mathbf{R})$.

- $\mathcal{C}_{0}^{\infty}(\mathbf{R}), \mathcal{D}(\mathbf{R})$ or $\mathcal{D}$. Let $\mathcal{C}_{0}^{\infty}(\mathbf{R})$ be the linear space of complex functions $\varphi \in \mathcal{E}$, with compact support in $\mathbf{R}$. Each function in this space is called a test function and such function constitute the base for the study of the modern distribution theory. (See [4], [7] ).

- $\mathcal{S}(\mathbf{R})$ or $\mathcal{S}$. A function $\varphi: \mathbf{R} \rightarrow \mathbf{C}$, is said to be of rapid descent at infinity, if $\varphi \in \mathcal{E}$ and

$$
\left|t^{m} \varphi^{(k)}(t)\right| \leq C_{m k},-\infty<t<\infty,
$$

where $m$ and $k$ run through all nonnegative integers, and $C_{m k}$ are constants, which only depends upon $m$ and $k$.

Let $\mathcal{S}(\mathbf{R})$ denote the linear subspace of $\mathcal{E}$ formed by those functions $\varphi$ that are rapidly descent at infinity. $\mathcal{S}(\mathbf{R})$ is called the $S$ chwartz's space and we will denote it by $\mathcal{S}$ instead of $\mathcal{S}(\mathbf{R})$.

It is well known that $\mathcal{S}$ is one of the best spaces where anyone can study the classical Fourier transform, as it is usually defined:

$$
\Lambda: \mathcal{S} \rightarrow \mathcal{S}, \varphi \mapsto \Lambda(\varphi)
$$

where,

$$
\Lambda(\varphi)(\xi)=\frac{1}{\sqrt{2 \pi}} \int_{-\infty}^{\infty} e^{-i t \xi} \varphi(t) d t
$$

and we call this function $\Lambda$ the Fourier transform operator, on $\mathcal{S}$. For details see $([2],[6])$, for instance.

\subsection{Linear Algebra}

Let $V$ be a complex vector space and denote by $V^{\star}$ the algebraic dual space of $V$, that is, the space of all linear functional defined on $V$. (See [5]). For example, if we have in mind the inclusions,

$$
\mathcal{D} \subseteq \mathcal{S} \subseteq \mathcal{E}
$$

then, after taking the algebraic dual operation, we obtain,

$$
\mathcal{E}^{\star} \subseteq \mathcal{S}^{\star} \subseteq \mathcal{D}^{\star}
$$


Now, if the complex vector space $V$ have an hermitian product, $\langle\cdot, \cdot\rangle$, then we can see $V^{\star}$ as an extension of $V$. In fact it is enough to consider the injective operator,

$$
j: V \rightarrow V^{\star}, v \mapsto j(v)=\langle\cdot, v\rangle,
$$

where $\langle\cdot, v\rangle$ denotes the linear functional on $V$, given by $w \mapsto\langle w, v\rangle$.

In the same direction let $V$ and $W$ be two vector spaces, over $\mathbf{C}$, endowed with their respective hermitian products, and let $L: V \rightarrow W$ be a linear operator. Suppose that there exist a linear operator $L^{+}: W \rightarrow V$ such that,

$$
\langle L v, w\rangle=\left\langle v, L^{+} w\right\rangle, \forall v \in V, \forall w \in W .
$$

The linear operator $L^{+}$is called the adjoint operator of $L$. Next, we can define the linear operator,

$$
\mathcal{L}: V^{\star} \rightarrow W^{\star}, \varphi \mapsto \mathcal{L}(\varphi)=\varphi \circ L^{+} .
$$

Since

$$
\mathcal{L}(\langle\cdot, v\rangle)(w)=\langle\cdot, v\rangle \circ L^{+}(w)=\left\langle L^{+} w, v\right\rangle=\langle w, L v\rangle,
$$

we conclude that the following diagram is commutative:

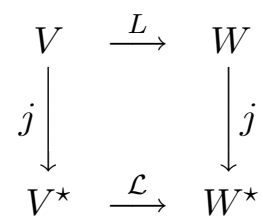

\section{A Problem of Eigenvalues and Eigenvectors}

It is known, from Classical Mechanics, that the position of a particle in the real line, as well as its momentum, at time $t$, are given by real numbers. But in Quantum Mechanics the state of a particle, at time $t$, is given by a function $\psi \in \mathcal{L}^{2}(\mathbf{R})$, such that $\int_{\mathbf{R}}|\psi|^{2}<\infty$. On the other hand, the observable operators, position and momentum, have the following interpretation: let $I \subset \mathbf{R}$ be an interval. Then $\int_{I}|\psi|^{2}$ is considered as the probability of founding the particle in that interval $I$, at time $t$, and $\int_{I}|\Lambda \psi|^{2}<\infty$ is the probability that this particle has a given momentum in the interval $I$, at time $t$, (see Section 5 for the extension of $\Lambda$ to $\mathcal{L}^{2}(\mathbf{R})$ ).

After this considerations we introduce both, the position operator and the momentum operator. See [3]. 
In fact they are the linear operators, defined respectively, by

$$
P: \mathcal{D} \rightarrow \mathcal{D}, \varphi \mapsto P \varphi, \text { with }(P \varphi)(x)=x \varphi(x), \forall x \in \mathbf{R},
$$

and

$$
M: \mathcal{S} \rightarrow \mathcal{S}, \varphi \mapsto M \varphi=-i \varphi^{\prime} .
$$

Note that, we also can choice $\mathcal{D}$ or $\mathcal{E}$ as the domain of both operators $M$ and $P$, it will depend on our purposes.

\subsection{The Eigenvalues of $\mathcal{P}$}

First of all we note that does not exist eigenvector solution, $\varphi \in \mathcal{D}$, of the equation,

$$
P \varphi=\lambda \varphi, \lambda \in \mathbf{R} .
$$

It seems strange because from Quantic Mechanics, the eigenvectors of $P$ represent the state of a particle in the position $\lambda$. In order to get around this difficulty we consider the extension operator $\mathcal{P}$, of $P$, as follow: first we compute the adjoint operator $P^{+}$, of $P$, under the hermitian product

$$
\langle f, g\rangle=\int_{-\infty}^{\infty} f(t) \overline{g(t)} d t, f, g \in \mathcal{D} .
$$

Now we observe that,

$$
\langle P \varphi, \psi\rangle=\int_{-\infty}^{\infty} x \varphi(x) \overline{\psi(x)} d x=\int_{-\infty}^{\infty} \varphi(x) \overline{x \psi(x)} d x=\langle\varphi, P \psi\rangle, \quad \forall \varphi, \psi \in \mathcal{D} .
$$

So the adjoint operator, $P^{+}$, of $P$, do exist and $P^{+}=P$. Thus according to (2.2) we get the extension $\mathcal{P}: \mathcal{D}^{\star} \rightarrow \mathcal{D}^{\star}$, given by $\mathcal{P} \varphi=\varphi \circ P^{+}=$ $\varphi \circ P$. After this we propose to study the following extended eigenvalueeigenvector equation,

$$
\mathcal{P}(T)=\lambda T, T \in \mathcal{D}^{\star}, \lambda \in \mathbf{C} .
$$

We begin by introducing the "object" delta of Dirac $\delta_{\lambda}, \lambda \in \mathbf{R}$. It is defined by: $\delta_{\lambda}(t)=\left\{\begin{aligned} 0 & \text {, if } t \neq \lambda \\ +\infty & \text {, if } t=\lambda\end{aligned}\right.$ and $\int_{-\infty}^{\infty} \delta_{\lambda}(t) d t=1$

Curiously this strange mathematical "object" works out very well in physical situations and in mathematics itself, although this "object" can not be considered as a function in the classical sense. In spite of this we 
often call it: delta function of Dirac, concentred in $\lambda$, or Dirac $\delta_{\lambda}$ function. The physicists regard the Dirac delta function $\delta_{\lambda}$ as a "state" in which the particle is localized at the point $\lambda \in \mathbf{R}$. Such "state" can be approximated by a regular state $\psi_{\epsilon} \in \mathcal{D}$, such that,

$$
\psi_{\epsilon}(t)=0 \text {, outside }[\lambda-\epsilon, \lambda+\epsilon] \text {, for small } \epsilon>0 \text {, and } \int \psi_{\epsilon}=1 \text {. }
$$

By tradition the Dirac delta function, concentred in zero, is denoted by $\delta$, that is $\delta_{0}=\delta$. More and much more deep information about the delta of Dirac can be found in [1]. Nowadays, via distribution theory, see [7], the Dirac delta function $\delta_{\lambda}$ is properly seen as a linear functional, and in this paper, we will define it in the following way:

$$
\begin{aligned}
\delta_{\lambda}: \mathcal{S} & \longrightarrow \mathbf{C} \\
\varphi & \longmapsto \delta_{\lambda}(\varphi)=\varphi(\lambda),
\end{aligned}
$$

so we have that $\delta_{\lambda}$ belongs to $\mathcal{S}^{\star}$.

3.1. Proposition. Consider the notation as in (3.4) and (3.5). Then we have:

$1 \lambda \in \mathbf{C}$ is an eigenvalue of $\mathcal{P}$ if and only if $\lambda \in \mathbf{R}$.

2 Let $E_{\lambda}(\mathcal{P}) \subset \mathcal{D}^{\star}$ be the eigenspace of $\mathcal{P}$, associated to $\lambda \in \mathbf{R}$. Then $E_{\lambda}(\mathcal{P})$ is generated by $\delta_{\lambda}$, that is $E_{\lambda}(\mathcal{P})=\left[\delta_{\lambda}\right]$.

Proof. First of all, if $\lambda$ (real or complex) is an eigenvalue of $\mathcal{P}$ with eigenvector $T \in \mathcal{D}^{\star}$, then $\mathcal{P}(T)=\lambda T$ and hence $(T \circ P) \varphi=\lambda T \varphi$, for all $\varphi \in \mathcal{D}$. It follows that,

$$
T((P-\lambda) \varphi)=0, \forall \varphi \in \mathcal{D} .
$$

1 . Suppose that $\lambda$ is a complex number having a non-zero imaginary part, and $\lambda$ is an eigenvalue of $\mathcal{P}$ with eigenvector $T \in \mathcal{D}^{\star}$.

We observe that the function $g(x)=\frac{1}{x-\lambda}$ belongs to $\mathcal{E}$ and $g \varphi \in \mathcal{D}$, if $\varphi \in \mathcal{D}$. Moreover $\varphi=(P-\lambda) g \varphi$. Therefore, by $(\star)$,

$$
T(\varphi)=T((P-\lambda) g \varphi)=0, \forall \varphi \in \mathcal{D} .
$$

Thus, $T \equiv 0$.

2. At first, we prove that 
$\mathcal{P}\left(\delta_{\lambda}\right)=\lambda \delta_{\lambda}, \lambda \in \mathbf{R}$. In fact: by definition we have, $\mathcal{P}\left(\delta_{\lambda}\right)=\delta_{\lambda} \circ P$. Hence,

$$
\left(\delta_{\lambda} \circ P\right)(\varphi)=\delta_{\lambda}(P \varphi)=P \varphi(\lambda)=\lambda \varphi(\lambda)=\lambda \delta_{\lambda}(\varphi), \forall \varphi \in \mathcal{D} .
$$

Therefore, $\mathcal{P}\left(\delta_{\lambda}\right)=\lambda \delta_{\lambda}$ and $\left[\delta_{\lambda}\right] \subset E_{\lambda}(\mathcal{P})$. Now let $T$ belongs to $\mathcal{D}^{\star}$ be an eigenvector of $\mathcal{P}$, associated to the eigenvalue $\lambda \in \mathbf{R}$.

Next one notes that if $\varphi \in \mathcal{D}$ and verify the condition $\varphi(\lambda)=0$, then we can find $\psi \in \mathcal{E}$ such that $\varphi=(P-\lambda) \psi$. In fact, if $\varphi$ belongs to $\mathcal{D}$ then we have that $\psi \in \mathcal{D}$, necessarily. Thus keeping in mind $(\star)$ this implies that

$$
T(\varphi)=0, \forall \varphi \in \mathcal{D} \text {, such that } \varphi(\lambda)=0 .
$$

In the general case we have,

$$
\varphi=\varphi(\lambda)+(P-\lambda) \psi
$$

for some $\psi \in \mathcal{E}$. Now let us consider $\theta \in \mathcal{D}$ such that $\theta(\lambda)=1$. We multiply the above equation by $\theta$ and we obtain:

$$
\theta \varphi=\varphi(\lambda) \theta+(P-\lambda)(\theta \psi)
$$

Note that as much $(1-\theta) \varphi$ as $\widetilde{\psi}=(P-\lambda)(\theta \psi)$ belong to $\mathcal{D}$ and vanish at $\lambda$. Thus, it follows from $(3.6)$ that $T((1-\theta) \varphi)=T(\widetilde{\psi})=0$. So we get: $\mathrm{T}(\varphi)=T((1-\theta) \varphi+\theta \varphi)=T(\theta \varphi)=\varphi(\lambda) T(\theta)+T(\widetilde{\psi})=T(\theta) \delta_{\lambda}(\varphi)$. We left to the reader the task of verifying that $T(\theta)=T\left(\theta_{1}\right)$, for every $\theta, \theta_{1} \in \mathcal{D}$, such that $\theta(\lambda)=\theta_{1}(\lambda)=1$.

Therefore $T=T(\theta) \delta_{\lambda}$ and this show us that $E_{\lambda}(\mathcal{P}) \subset\left[\delta_{\lambda}\right]$ and conclude the proposition.

\subsection{The Eigenvalues of $\mathcal{M}$}

As we have observed, we can consider the momentum operator as having domain $\mathcal{D}, \mathcal{S}$ or $\mathcal{E}$. In this paper, when we defined this operator, we have made the option by $\mathcal{S}$ to be the domain of $M$ and next we show to the reader that this is the best choice from the physical point of view.

In any case if we consider, $\mathcal{D}$ or $\mathcal{S}$, as the domain of $M$, then we use the hermitian product as defined in (3.3). It is not difficult to show that, $M^{+}$, the adjoint of $M$, exist and is equal to $M$. Thus, we can consider the extension, $\mathcal{M}$, of $M$, defined over $\mathcal{D}$ or $\mathcal{S}$. Of course, $\mathcal{M}(\varphi)=\varphi \circ M$, according to (2.2). 
In order to study the eigenvalue-eigenvector problem with respect to the operator $\mathcal{M}$, we begin noting that the map

$$
\begin{aligned}
\mathcal{E} \times \mathcal{D}^{\star} & \longrightarrow \mathcal{D}^{\star} \\
(f, T) & \longmapsto f \cdot T,
\end{aligned}
$$

where $f \cdot T(\varphi)=T(f \varphi)$, for every $\varphi \in \mathcal{D}$, turns $\mathcal{D}^{\star}$ into an $\mathcal{E}$-module. As a consequence the following equation works out:

$$
\mathcal{M}(f \cdot T)=M(f) \cdot T+f \cdot \mathcal{M}(T), \forall f \in \mathcal{E}, T \in \mathcal{D}^{\star} .
$$

After (3.7) it is easy to see that $e^{i \lambda x} T$ belongs to the kernel of $\mathcal{M}$.

Over and above that, in an injective way, for each $f \in \mathcal{E}$ we associate, $T_{f} \in \mathcal{D}^{\star}$, defined by,

$$
T_{f}(\varphi)=\int \varphi f, \forall \varphi \in \mathcal{D} .
$$

Thus, we identify each function $f \in \mathcal{E}$ with $T_{f} \in \mathcal{D}^{\star}$.

For example, for each $\lambda \in \mathbf{C}$, the function $f_{\lambda}(x)=e^{i \lambda x}, x \in \mathbf{R}$, is such that, $f_{\lambda} \in \mathcal{E}$ and $M\left(f_{\lambda}\right)=\lambda f_{\lambda}$. Having in mind this identification and the definition of $\mathcal{M}$, we obtain $\mathcal{M}\left(T_{f_{\lambda}}\right)=\lambda T_{f_{\lambda}}$, for each $\lambda \in \mathbf{C}$ and $f_{\lambda}(x)=e^{i \lambda x}$.

In fact,

$$
\mathcal{M}\left(T_{f_{\lambda}}\right)(\varphi)=T_{f_{\lambda}}\left(-i \varphi^{\prime}\right)=\int-i \varphi^{\prime} e^{i \lambda x}=\lambda \int \varphi e^{i \lambda x}=\lambda T_{f_{\lambda}} \varphi, \forall \varphi \in \mathcal{D} .
$$

Now, with a focus on the next proposition, we present an auxiliary lemma which describe the kernel of $\mathcal{M}$.

3.2. Lemma. $\mathcal{M}(T)=0$ if and only if $T \in\left[T_{\widetilde{1}}\right]$.

Proof. First, remember that $T_{\widetilde{1}} \in \mathcal{D}^{\star}$, is the functional identified with $\widetilde{1} \in \mathcal{E}$.

We only prove the direct implication. So let $T \in \mathcal{D}^{\star}$, such that $\mathcal{M}(T)=0$. Then $T(M \varphi)=0, \forall \varphi \in \mathcal{D}$. Thence $T \varphi^{\prime}=0$, for every $\varphi \in \mathcal{D}$. It is well known that if $\varphi \in \mathcal{D}$, then there exist $\psi \in \mathcal{D}$ such that $\varphi=\psi^{\prime}$, if and only if $\int_{-\infty}^{\infty} \varphi(t) d t=0$.

So let us take $\theta \in \mathcal{D}$ such that $\int_{-\infty}^{\infty} \theta(x) d x=1$ and, for each $\varphi \in \mathcal{D}$, consider 
the function $\widetilde{\varphi}=\varphi-\left[\int_{-\infty}^{\infty} \varphi(u) d u\right] \theta$. So $\widetilde{\varphi} \in \mathcal{D}$, and $\int_{-\infty}^{\infty} \widetilde{\varphi}(u) d u=0$. Then there exist $\psi \in \mathcal{D}$, such that $\psi^{\prime}=\widetilde{\varphi}$. Thus $T(\widetilde{\varphi})=T\left(\psi^{\prime}\right)=0$. It implies $T \varphi=\left[\int_{-\infty}^{\infty} \varphi(u) d u\right] T(\theta)=T(\theta) \widetilde{1}(\varphi)=T(\theta) T_{\widetilde{1}}(\varphi)$, for every $\varphi \in \mathcal{D}$. Therefore, if $\mathcal{M}(T)=0$, then $T=c T_{\widetilde{1}}$, for some constant $c$. In fact this constant $c$ is independent of the choice of $\theta \in \mathcal{D}$, such that $\int \theta=1$.

3.3. Proposition. For each complex number $\lambda$, let $E_{\lambda}(\mathcal{M})$ be the eigenspace associated to the eigenvalue $\lambda$, of $\mathcal{M}$.Then,

$1 E_{\lambda}(\mathcal{M})=\left[T_{f_{\lambda}}\right]$, where $f_{\lambda}(x)=e^{i \lambda x}$.

$2 T_{f_{\lambda}} \in \mathcal{S}^{\star}$ if and only if $\lambda \in \mathbf{R}$.

Proof. 1 We have already proved the inclusion $\left[T_{f_{\lambda}}\right] \subset E_{\lambda}(\mathcal{M})$. To prove the opposite inclusion, we observe that $\mathcal{M}\left(f_{-\lambda} T\right)=0$. Then by Lemma 3.2, we get $f_{-\lambda} T=\mu T_{\widetilde{1}}$ for some constant $\mu \in \mathbf{C}$. Thus, $T=\mu f_{\lambda} T_{\widetilde{1}}=\mu T_{f_{\lambda}}$.

2 Let $\lambda=a+i b \in \mathbf{C}$, such that $T_{f_{\lambda}} \in \mathcal{S}^{\star}$ and suppose that $b \neq 0$. Let us take $b>0$, (the case $b<0$ is left to the reader). Thus $f_{\lambda}(x)=e^{i \lambda x}=$ $e^{-b x} e^{i a x}$. Next we are going consider the following function:

$$
\psi(x)=\left\{\begin{array}{ccc}
e^{b x} & \text { if } & x \leq-1 \\
\theta(x) & \text { if } & -1 \leq x \leq 1 \\
0 & \text { if } & x \geq 1
\end{array}\right.
$$

where $\theta$ is a $\mathcal{C}^{\infty}$-function, defined in $[-1,1]$, that yields $\psi$ in such a way that $\psi$ belongs to $\mathcal{S}$. Now we can consider the function $\varphi=\psi e^{-i a x}, x \in \mathbf{R}$. We have that $\varphi \in \mathcal{S}$ and

$$
T_{f_{\lambda}}(\varphi)=\int_{-\infty}^{\infty} f_{\lambda}(x) \varphi(x) d x=\int_{-\infty}^{-1} d x+\int_{-1}^{1} \theta(x) e^{-b x} d x=\infty,
$$

and this implies that $T_{f_{\lambda}}$ it is a not well defined functional over $\mathcal{S}$, that is $T_{f_{\lambda}} \notin \mathcal{S}^{\star}$ and the Lemma is proved.

\section{Fourier's Inverse Transform}

First let us consider the operator,

$$
I: \mathcal{S} \rightarrow \mathcal{S}, \varphi \mapsto I(\varphi) \text {, where } I(\varphi)(x)=\varphi(-x)
$$


We have in mind to prove the equation,

$$
\Lambda^{2}=I
$$

where $\Lambda$ denotes the Fourier transform operator. In order to do that we need some auxiliary results.

4.1. Lemma. Let $P, Q \in \mathcal{L}(\mathcal{S})$. Then $P=Q$ if and only if $\delta_{\lambda} \circ P=\delta_{\lambda} \circ Q$, for all $\lambda \in \mathbf{R}$.

Proof. Assume $\delta_{\lambda} \circ P=\delta_{\lambda} \circ Q$, for all $\lambda \in \mathbf{R}$. Then, $\left(\delta_{\lambda} \circ P\right) \varphi=$ $\left(\delta_{\lambda} \circ Q\right) \varphi, \forall \varphi \in \mathcal{S}$ or yet,

$$
(P \varphi)(\lambda)=(Q \varphi)(\lambda), \forall \lambda \in \mathbf{R}, \forall \varphi \in \mathcal{S} .
$$

Therefore $P=Q$.

Now, for each $\lambda \in \mathbf{R}$ we introduce the translation operator by $\lambda$ :

$$
\tau_{\lambda}: \mathcal{S} \rightarrow \mathcal{S}, \varphi \mapsto \tau_{\lambda}(\varphi)
$$

where $\tau_{\lambda}(\varphi)(x)=\varphi(x-\lambda)$.

4.2. Lemma. Let $P, Q \in \mathcal{L}(\mathcal{S})$ and assume that,

$$
P \circ \tau_{\lambda}=\tau_{-\lambda} \circ P, Q \circ \tau_{\lambda}=\tau_{-\lambda} \circ Q, \forall \lambda \in \mathbf{R},
$$

and

$$
\delta \circ P=\delta \circ Q
$$

Then $P=Q$.

Proof. From (4.4) we have $\delta \circ P \circ \tau_{\lambda}=\delta \circ Q \circ \tau_{\lambda}$. Now we apply (4.3) to get, $\delta \circ \tau_{-\lambda} \circ P=\delta \circ \tau_{-\lambda} \circ Q, \forall \lambda \in \mathbf{R}$. But,

$$
\left(\delta \circ \tau_{-\lambda}\right) \varphi=\delta\left(\tau_{-\lambda} \varphi\right)=\left(\tau_{-\lambda} \varphi\right)(0)=\varphi(\lambda)=\delta_{\lambda}(\varphi), \forall \varphi \in \mathcal{S} .
$$

Therefore, $\delta \circ \tau_{-\lambda}=\delta_{\lambda}$ and $\delta_{\lambda} \circ P=\delta_{\lambda} \circ Q$, for all $\lambda \in \mathbf{R}$. By Lemma 4.1 it follows that $P=Q$.

4.3. Lemma. For each $\lambda \in \mathbf{R}$, let $m_{\lambda}: \mathcal{S} \rightarrow \mathcal{S}$ be the multiplication operator defined by: $m_{\lambda}(\varphi)(\xi)=e^{-i \lambda \xi} \varphi(\xi)$. Then, $\Lambda \circ \tau_{\lambda}=m_{\lambda} \circ \Lambda$ and $\Lambda \circ$ $m_{\lambda}=\tau_{-\lambda} \circ \Lambda$. 
Proof. Straightforward.

4.4. Lemma. Notations as in (2.1), (3.1) and (3.2). Then, $\Lambda \circ P=$ $-M \circ \Lambda$ and $\Lambda \circ M=P \circ \Lambda$.

Proof. In fact,

$$
\begin{aligned}
{[(\Lambda \circ P)(\varphi)](\xi) } & =\Lambda(P \varphi)(\xi)=\frac{1}{\sqrt{2 \pi}} \int_{-\infty}^{\infty} e^{-i x \xi}(P \varphi)(x) d x \\
& =\frac{i}{\sqrt{2 \pi}} \int_{-\infty}^{\infty} e^{-i x \xi}(-i x) \varphi(x) d x \\
& =\frac{i}{\sqrt{2 \pi}} \int_{-\infty}^{\infty} \frac{\partial}{\partial \xi} e^{-i x \xi} \varphi(x) d x \\
& =i \frac{\partial}{\partial \xi}(\Lambda \varphi)(\xi)=\left(i \frac{\partial}{\partial \xi} \circ \Lambda\right)(\varphi)(\xi)=-[(M \circ \Lambda)(\varphi)](\xi) .
\end{aligned}
$$

Next we prove that $\Lambda \circ M=P \circ \Lambda$. We have,

$$
\begin{aligned}
{[(\Lambda \circ M) \varphi](\xi) } & =\Lambda(M \varphi)(\xi)=\frac{1}{\sqrt{2 \pi}} \int_{-\infty}^{\infty} e^{-i x \xi}(M \varphi)(x) d x \\
& =\frac{1}{\sqrt{2 \pi}} \int_{-\infty}^{\infty} e^{-i x \xi}\left(-i \frac{\partial \varphi}{\partial x}(x)\right) d x \\
& =\frac{-i}{\sqrt{2 \pi}} \int_{-\infty}^{\infty} e^{-i x \xi} \frac{\partial \varphi}{\partial x}(x) d x \\
& =\frac{\xi}{\sqrt{2 \pi}} \int_{-\infty}^{\infty} e^{-i x \xi} \varphi(x) d x=\xi \Lambda \varphi(\xi)=(P \circ \Lambda)(\varphi)(\xi)
\end{aligned}
$$

Now let us come back to the equation, $\Lambda^{2}=I$, in (4.2). For this we study the operator $\delta \circ \Lambda^{2}$ in the next Proposition.

4.5. Proposition. $\delta \circ \Lambda^{2}=\delta$.

Proof. First of all, we prove that $\delta \circ \Lambda^{2}$ belongs to kernel of $\mathcal{P}$. In fact: we know that $\mathcal{P}(T)=T \circ P$, for all $T \in \mathcal{D}^{\star}$.

Then we must prove that $\mathcal{P}\left(\delta \circ \Lambda^{2}\right)=0$. But from Lemma 4.4 we have: $\delta \circ \Lambda^{2} \circ P=\delta \circ \Lambda \circ \Lambda \circ P=\delta \circ \Lambda \circ(-(M \circ \Lambda))=\delta \circ(-P) \circ \Lambda^{2}$.

On the other hand, $\delta \circ(-P) \circ \Lambda^{2}=0$, thus

$$
\mathcal{P}\left(\delta \circ \Lambda^{2}\right)=\delta \circ \Lambda^{2} \circ P=\delta \circ(-P) \circ \Lambda^{2}=0,
$$


and $\delta \circ \Lambda^{2} \in \operatorname{ker}(\mathcal{P})$. As $\operatorname{ker}(\mathcal{P})=[\delta]$, we have

$$
\delta \circ \Lambda^{2}=c \delta, \text { for some constant } c .
$$

Next we prove that $c=1$. Well, evaluating (4.5), at $\phi_{0} \in \mathcal{S}, \phi_{0}(t)=$ $e^{-\frac{t^{2}}{2}}, t \in \mathbf{R}$, and using that $\phi_{0}$ is an eigenvector, of $\Lambda$, associated to the eigenvalue $\lambda=1$, it follows that,

$$
\left(\delta \circ \Lambda^{2}\right) \phi_{0}=(\delta \circ \Lambda)\left(\Lambda\left(\phi_{0}\right)\right)=(\delta \circ \Lambda)\left(\phi_{0}\right)=\delta\left(\phi_{0}\right)=1=c=c \delta\left(\phi_{0}\right) .
$$

Therefore $\delta \circ \Lambda^{2}=\delta$.

\subsection{Corollary. $\Lambda^{2}=I$.}

Proof. We can see, without difficulties, that $\delta \circ I=\delta$. So it follows from Proposition 4.5 that $\delta \circ \Lambda^{2}=\delta=\delta \circ I$. On the other hand it is easy to verify that

$$
I \circ \tau_{\lambda}=\tau_{-\lambda} \circ I, \forall \lambda \in \mathbf{R} .
$$

Moreover follows from Lemma 4.2 that

$$
\Lambda^{2} \circ \tau_{\lambda}=\tau_{-\lambda} \circ \Lambda^{2}, \forall \lambda \in \mathbf{R} .
$$

Thus keeping in mind (4.6) and (4.7), we conclude from Lemma 4.3 that $\Lambda^{2}=I$.

4.7. Theorem. There exist the Fourier's inverse transform of $\Lambda$ and $\Lambda^{-1}=\Lambda^{3}$.

Proof. After Corollary 4.1 , we have $\Lambda^{4}=1_{\mathcal{S}}$, where $1_{\mathcal{S}}$ is the identity operator in $\mathcal{S}$. Therefore $\Lambda^{3}=\Lambda^{-1}$ is the Fourier's inverse transform, as we wanted!

Here we register the pioneer idea from A. Calderón ${ }^{1}$ that use the solution space of the ordinary differential equation

$$
y^{\prime}+t y=0
$$

to show that $\phi_{0}(t)=e^{-\frac{t^{2}}{2}}$ is an eigenvector of the Fourier transform $\Lambda$. Moreover, this computation reveal us a fine application of Quantum Mechanics in order to determine $\Lambda \phi_{0}$.

\footnotetext{
${ }^{1}$ Alberto Calderón (1920 - 1998). Was born in Argentina. One of the most influential mathematicians of the 20 th century.
} 
It is well known that the solution space of (4.8) has dimension one and that space is generated by the function $\phi_{0}(t)=e^{-\frac{t^{2}}{2}}$. Having in mind that $M \varphi=-i \varphi^{\prime}$ and $P \varphi(x)=x \varphi(x)$, the equation (4.8) can be rewrite in the form

$$
i M \phi+P \phi=0 .
$$

If we apply Fourier transform, from (4.9), we get, i $\Lambda M \phi+\Lambda P \phi=0$.

Then, by Lemma 4.4, we have:

$$
i M(\Lambda \phi)+P(\Lambda \phi)=0 .
$$

By the fact solution space of (4.8) has dimension one, and that it is generated by $\phi_{0}(t)$, we have,

$$
\Lambda \phi_{0}(t)=c \phi_{0}(t), \text { for some constant } c .
$$

Moreover, $\Lambda \phi_{0}(0)=c=1$.

\section{Fourier Transforms}

All the time in this paper, we have spoken about "the" Fourier transform. Under certain point of view this is not so correct. In fact we can say that there are several Fourier transforms, as we now explain.

At first we introduce the spaces, $\mathcal{L}^{1}(\mathbf{R})$ and $\mathcal{L}^{2}(\mathbf{R})$. (See [2]).

$\mathcal{L}^{1}(\mathbf{R})$ is the space of (class of) mesurable functions $f: \mathbf{R} \rightarrow \mathbf{C}$, such that $|f|$ has finite Lebesgue integral over $\mathbf{R} . \mathcal{L}^{1}(\mathbf{R})$ is a Banach space.

$\mathcal{L}^{2}(\mathbf{R})$ denotes the space of (class of) mesurable functions $f: \mathbf{R} \rightarrow \mathbf{C}$, such that $|f|^{2}$ has finite Lebesgue integral over $\mathbf{R} . \mathcal{L}^{2}(\mathbf{R})$ is a vector space and if $f, g \in \mathcal{L}^{2}(\mathbf{R})$ we define an hermitian product and a norm,

$$
(f, g)=\int_{-\infty}^{\infty} f(x) \overline{g(x)} d x ; \quad|f|=\left(\int_{-\infty}^{\infty}|f(x)|^{2} d x\right)^{1 / 2}, \text { respectively }
$$

With respect to this norm $\mathcal{L}^{2}(\mathbf{R})$ is a complete metric space, that is, a Hilbert space, also called space of square integrable functions. Note that $\mathcal{S} \subset \mathcal{L}^{1}(\mathbf{R}) \cap \mathcal{L}^{2}(\mathbf{R})$ and $\mathcal{S}$ is dense in $\mathcal{L}^{2}(\mathbf{R})$. 


\subsection{Fourier Transform in $\mathcal{L}^{1}(\mathbf{R})$}

If we use the rule in $(2.1)$ we can associate to each function $f \in \mathcal{L}^{1}(\mathbf{R})$ the function $\Lambda f$, with $(\Lambda f)(\xi)=\frac{1}{\sqrt{2 \pi}} \int_{-\infty}^{\infty} f(x) e^{-i \xi x} d x$.

After some computations it can be verified that $\Lambda f \in \mathcal{C}_{\infty}^{0}(\mathbf{R})$, the space of continuous functions $f: \mathbf{R} \rightarrow \mathbf{C}$, such that $\lim _{|x| \rightarrow \pm \infty} f(x)=0$. Thus we have the Fourier transform in $\mathcal{L}^{1}(\mathbf{R})$, given by $\Lambda: \mathcal{L}^{1}(\mathbf{R}) \rightarrow \mathcal{C}_{\infty}^{0}(\mathbf{R})$. For details see [2].

\subsection{Fourier Transform in $\mathcal{L}^{2}(\mathbf{R})$}

$\mathcal{L}^{2}(\mathbf{R})$ is a very good space to localize the observable, position and momentum, in Quantum Mechanics. In despite of this we have some problems. For example, the Fourier transform for $\mathcal{L}^{2}$-functions cannot be defined according to (2.1). However, it is possible to overcome this difficulty by invoking the density of $\mathcal{S}$ in $\mathcal{L}^{2}(\mathbf{R})$. If $f \in \mathcal{L}^{2}(\mathbf{R})$, there exist a sequence $\left(f_{n}\right)_{n}, f_{n} \in \mathcal{S}, \forall n \in \mathbf{N}$, such that

$$
f_{n} \rightarrow f, \text { in } \mathcal{L}^{2}(\mathbf{R}) .
$$

Since $\left(\Lambda f_{n}\right)_{n}$ is a Cauchy sequence in the complete metric space $\mathcal{L}^{2}(\mathbf{R})$ it converges to some $F \in \mathcal{L}^{2}(\mathbf{R})$. Thus it makes sense to define,

$$
\widetilde{\Lambda}: \mathcal{L}^{2}(\mathbf{R}) \longrightarrow \mathcal{L}^{2}(\mathbf{R}), f \longmapsto \widetilde{\Lambda} f,(\widetilde{\Lambda} f)(\xi)=\lim _{n \rightarrow \infty} \Lambda f_{n}=F,
$$

and call it the Fourier transform in $\mathcal{L}^{2}(\mathbf{R})$. In fact, it is very simple to prove that the Fourier transform, for $\mathcal{L}^{2}$-functions, is a very well defined linear operator. But the Fourier transform of a square integrable function it is not punctually defined, unlike the Fourier transform of integrable functions. Thus we can say that the $\mathcal{L}^{2}$-Fourier transform is one another Fourier transform. For details see [6].

\subsection{Fourier Transform in $\mathcal{S}^{\star}$}

It is easy to show that the adjoint operator, $\Lambda^{+}$, of the Fourier transform $\Lambda: \mathcal{S} \rightarrow \mathcal{S}$, defined in (2.1), do there exist and it is equal to $\Lambda^{3}$, making it clear,

$$
\left(\Lambda^{+} \varphi\right)(\xi)=\frac{1}{\sqrt{2 \pi}} \int_{-\infty}^{\infty} e^{i x \xi} \varphi(x) d x .
$$

So, according to $(2.2)$ we can consider the generalized Fourier transform $\mathcal{F}: \mathcal{S}^{\star} \rightarrow \mathcal{S}^{\star}$, given by $\mathcal{F}(T)=T \circ \Lambda^{+}=T \circ \Lambda^{3}$. 
Next we observe that the hermitian product in $\mathcal{L}^{2}(\mathbf{R})$, allow us to identify the function $f \in \mathcal{L}^{2}(\mathbf{R})$, with the functional $\langle\cdot, f\rangle \in \mathcal{S}^{\star}$. In particular $\widetilde{\Lambda} f \in \mathcal{L}^{2}(\mathbf{R})$ can be identified with $\langle\cdot, \widetilde{\Lambda} f\rangle \in \mathcal{S}^{\star}$. Moreover it can be showed that

$$
\mathcal{F}(\langle\cdot, f\rangle)=\langle\cdot, \widetilde{\Lambda} f\rangle, \forall f \in \mathcal{L}^{2}(\mathbf{R}) .
$$

So, under identification, we have $\mathcal{F}(f)=\widetilde{\Lambda} f$, for every $f \in \mathcal{L}^{2}(\mathbf{R})$.

It is worthwhile to note that there exist functions that does not belong to

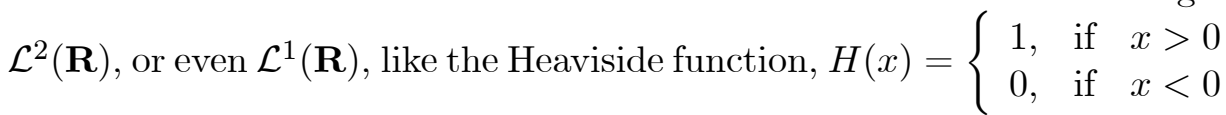
but in spite of this, it is possible to compute their generalized Fourier transform. For example, under the identification of the Heaviside function, $H$, with $\langle\cdot, H\rangle \in \mathcal{S}^{\star}$, we have

$$
\mathcal{F}(H)=\frac{i}{\sqrt{2 \pi}}\left\{\frac{1}{x}\right\}+\frac{\sqrt{2 \pi}}{2} \delta,
$$

where $\left\{\frac{1}{x}\right\}$ is the Cauchy principal value distribution. In fact, it can be defined by

$$
\left\{\frac{1}{x}\right\}(\varphi)=\left\{\begin{array}{ccc}
0 & \text { if } & \varphi \text { is even } \\
\int \frac{\varphi(x)}{x} d x & \text { if } & \varphi \text { is odd }
\end{array} .\right.
$$

Let us consider the Fourier transform of functions defined in $\mathbf{R}^{n}$, that is, we take functions $\varphi: \mathbf{R}^{n} \rightarrow \mathbf{C}$ in the space $\mathcal{S}\left(\mathbf{R}^{n}\right)$ and calculate its Fourier transform by defining:

$$
(\Lambda \varphi)(\xi)=\frac{1}{(2 \pi)^{n / 2}} \int e^{-i\langle\xi, x\rangle} \varphi(x) d x,
$$

where $\langle\cdot, \cdot\rangle$ denotes the inner product in $\mathbf{R}^{n}$. See [6] for details.

As we have made in Section 4 , it is possible to prove that $\Lambda^{2}=I_{\mathcal{S}\left(\mathbf{R}^{n}\right)}$. Here $\Lambda$ is the operator we have defined in (5.1) and $I(x)=-x$, for every $x \in \mathbf{R}^{n}$. In order to do this, we list some changes that have to be considered and left the verifications to the reader.

- The previous position and momentum operators must be replaced for $P_{k}, M_{k}, k=1, \ldots, n$, where $\left(P_{k} \varphi\right)(x)=x_{k} \varphi, M_{k} \varphi=-i \partial_{k} \varphi, x=$ $\left(x_{1}, \ldots, x_{n}\right)$ and $\partial_{k}=\frac{\partial}{\partial x_{k}}$, for each $\varphi \in \mathcal{S}\left(\mathbf{R}^{n}\right)$.

- Lemma 4.4 becomes $\Lambda \circ P_{k}=-M_{k} \circ \Lambda$ and $\Lambda \circ M_{k}=P_{k} \circ \Lambda$, for each $k \in\{1, \ldots, n\}$. 
- The key fact $E_{0}(\mathcal{P})=[\delta]$, in Proposition 3.1 that we have used to prove that $\Lambda^{4}=I$, now correspond to

$$
\bigcap_{k=1}^{n} E_{0}\left(\mathcal{P}_{k}\right)=[\delta]
$$

with $\mathcal{P}_{k}(T)=T \circ P_{k}, k=1, \ldots, n$. It is not difficult to prove this equality. We prove the inclusion

$$
\bigcap_{k=1}^{n} E_{0}\left(\mathcal{P}_{k}\right) \subset[\delta] .
$$

Let $T \in \bigcap_{k=1}^{n} E_{0}\left(\mathcal{P}_{k}\right)$ and $\varphi \in \mathcal{S}\left(\mathbf{R}^{n}\right)$. We choose $\psi_{k} \in \mathcal{E}\left(\mathbf{R}^{n}\right)$, such that

$$
\varphi=\varphi(0)+\sum_{k=1}^{n} \mathcal{P}_{k}\left(\psi_{k}\right)
$$

Next, take $\theta_{\varphi} \in \mathcal{C}_{0}^{\infty}\left(\mathbf{R}^{n}\right)$ such that $\theta_{\varphi}(x)=1$, for all $x \in \operatorname{supp}(\varphi) \cup$ $\{0\}$.

We have,

$$
\varphi=\varphi \theta_{\varphi}+\sum_{k=1}^{n} \mathcal{P}_{k}\left(\psi_{k} \theta_{\varphi}\right)
$$

Then,

$$
T(\varphi)=\varphi(0) T\left(\theta_{\varphi}\right)+\sum_{k=1}^{n} \mathcal{P}_{k}(T)\left(\psi_{k} \theta_{\varphi}\right)=T\left(\theta_{\varphi}\right) \varphi(0)=T\left(\theta_{\varphi}\right) \delta(\varphi) .
$$

It is crucial to understand that the constant $T\left(\theta_{\varphi}\right)$ is independent of $\varphi$, indeed!

- The function $\phi_{0}(x)=e^{-\frac{|x|^{2}}{2}},|x|^{2}=\sum_{k=1}^{n} x_{k}^{2}$, is an eigenvector of $\Lambda$ associated to eigenvalue $\lambda=1$.

\section{References}

[1] P. Cordaro and A. Kawano, O Delta de Dirac. Livraria da Física Editora, (2002). 
[2] D. G. Figueiredo, Análise de Fourier e Equações Diferenciais Parciais. Projeto Euclides, IMPA, (2000).

[3] J. Glimm and A. Jaffe, Quantum Physics, A Functional Integral Point of View. Springer, 2nd edition, (1987).

[4] J. Hounie, Teoria Elementar das Distribuições. IMPA, Rio de Janeiro, (1979).

[5] E. L. Lima, Álgebra Linear. IMPA, Segunda Edição, (1996).

[6] E. H. Lieb and M.Loss, Analysis. AMS, (1997).

[7] A. H. Zemanian, Distribution Theory and Transform Analysis: An Introduction to Generalized Functions, with Applications. Dover Publications, (2010).

\section{Nelson N. de O. Castro}

CCEN - Departamento de Matemática

Universidade Federal de Paraíba

Cidade Universitária - João Pessoa - PB

CEP : 58051-900

Brasil

e-mail : nelson@ccen.ufpb.br

\section{Ramón Mendoza}

CCEN - Departamento de Matemática

Universidad Federal de Pernambuco

Cidade Universitária

50740-540, Recife - PE

Brasil

e-mail : ramon@dmat.ufpe.br

and

\section{Jacqueline Rojas}

CCEN - Departamento de Matemática

Universidade Federal de Paraíba

Cidade Universitária - João Pessoa - PB

CEP : 58051-900

Brasil

e-mail : jacq@mat.ufpb.br 